\title{
PROPRIEDADES FÍSICAS DO SOLO E CRESCIMENTO DE RAÍZES DE MILHO EM UM ARGISSOLO VERMELHO SOB TRÁFEGO CONTROLADO DE MÁQUINAS ${ }^{(1)}$
}

\author{
Michael Mazurana ${ }^{(2)}$, Jessé Rodrigo Fink ${ }^{(2)}$, Victor Hugo da Silveira ${ }^{(3)}, \operatorname{Renato}_{\text {Levien }}^{(4)}$, \\ Lucas Zulpo $^{(5)}$ \& Darlan Brezolin ${ }^{(5)}$
}

\begin{abstract}
RESUMO
O desempenho das funções do solo pode ser influenciado pela compactação imposta pelo manejo inadequado. Algumas propriedades físicas do solo são tomados como indicadores da presença de camadas compactadas, as quais podem interferir no desenvolvimento radicular de culturas. Práticas agrícolas, como o uso de plantas de cobertura, que melhoram as propriedades do solo, podem mitigar problemas relacionados à compactação. $O$ objetivo deste trabalho foi avaliar propriedades físicas de solo e de plantas, estas cultivadas em condições controladas. Em um Argissolo Vermelho sob sistema plantio direto consolidado com dois manejos de cobertura de inverno (pousio e aveia + ervilhaca) e tráfego controlado de máquinas [sem tráfego (ST), antes do tráfego (AT) e depois do tráfego (DT)], avaliaram-se a pressão de pré-consolidação, a resistência do solo à penetração, a densidade do solo, a porosidade e o diâmetro médio ponderado de agregados. Amostras indeformadas de solo das condições antes e depois do tráfego foram coletadas em vasos de PVC e acondicionadas em casa de vegetação, nos quais foram semeadas três sementes de milho, e cultivadas durante 25 dias sob diferentes condições de disponibilidade hídrica. Após esse período, determinaram-se a área foliar, massa verde e seca da parte aérea e massa úmida e seca das raízes de plantas de milho. A densidade do solo independentemente da camada avaliada não foi influenciada pela condição de cobertura do solo, mas sim pela condição de tráfego. Já a macroporosidade foi influenciada pelo tráfego no sistema pousio até $0,10 \mathrm{~m}$, indicando ter esse sistema menor capacidade de suportar perturbações, comprovado pelos menores valores de pressão de pré-consolidação. A resistência do solo à penetração aumentou em profundidade, estando relacionada à maior densidade, menor
\end{abstract}

(1) Trabalho apresentado como um dos requisitos da disciplina Relação Solo-Planta, Programa de Pós-Graduação em Ciência do Solo da Universidade Federal do Rio Grande do Sul (PPGS/UFRGS). Recebido para publicação em 20 de junho de 2012 e aprovado em 24 de maio de 2013.

(2) Doutorando do Curso de Pós-Graduação em Ciência do Solo, UFRGS. Av. Bento Gonçalves, 7712. CEP $91540-000$ Porto Alegre (RS). Bolsista CNPq. E-mail: michael.mazurana@gmail.com; fink1j@gmail.com

(3) Mestrando do Curso de Pós-Graduação em Ciência do Solo, UFRGS. Bolsista CNPq. E-mail: vhsilver@ig.com.br

(4) Professor Associado, Departamento de Solos e Faculdade de Agronomia, UFRGS. E-mail: renatole@ufrgs.br

(5) Bolsistas de Iniciação Científica, UFRGS. E-mail: lucaszulpo@yahoo.com.br; darlanbrezolin@hotmail.com 


\begin{abstract}
macroporosidade e maior pressão de pré-consolidação. Sem restrição hídrica, o crescimento radicular do milho foi influenciado positivamente pelo tráfego de máquinas. Nas condições da experimentação, o plantio direto com sistema de rotação de culturas apresentou maior resiliência frente às perturbações do meio.
\end{abstract}

Termos de indexação: resistência à penetração, densidade do solo, capacidade de suporte de carga, massa de raízes.

\title{
SUMMARY: SOIL PHYSICAL PROPERTIES AND MAIZE ROOT GROWTH IN AN ULTISOL UNDER CONTROLLED MACHINE TRAFFIC
}

\begin{abstract}
Soil functions can be hampered by compaction induced by inadequate management. Some soil physical properties are indicators of the existence of compacted layers that can affect the root development of crops. Agricultural practices, e.g., planting of cover crops to improve soil properties, can mitigate the compaction-related problems. The aim of this study was to evaluate the physical attributes of soil and plants, grown under controlled conditions. In an Ultisol under no-tillage with two winter cover crops (fallow and oat + vetch) and controlled machine traffic (no traffic-NT, before traffic - BT and after traffic - AT), we evaluated the preconsolidation pressure, penetration resistance, soil bulk density, porosity, and average diameter of aggregates. Undisturbed soil samples were collected before and after traffic, filled into PVC pots and placed in a greenhouse. Three corn seeds were sown per pot and grown for 25 days under different conditions of water availability. After this period, the leaf area and the fresh and dry matter of corn shoots and roots were determined. Regardless of the layer, bulk density was not affected by the soil cover, but was influenced by machine traffic. Macroporosity in the fallow plot was affected by traffic to a depth of $0.10 \mathrm{~m}$, indicating the reduced capacity of this system to resist disturbances, evidenced also by the lower pre-consolidation pressure. Resistance to penetration increased with depth, and was related to higher density, lower macroporosity and higher pre-consolidation pressure. Without water stress, maize root growth was positively influenced by machine traffic. Under the tested experimental conditions, no-tillage with crop rotation was more resilient to soil disturbances than the other system.
\end{abstract}

Index terms: penetration resistance, bulk density, load bearing capacity, root mass.

\section{INTRODUÇÃO}

A compactação do solo tem sido apontada como uma das principais causas da degradação física de solos agrícolas. Isolada ou associada à degradação biológica e química, leva à redução da qualidade do solo, conceituada por Karlen et al. (1997) como a capacidade desse em desempenhar ou não suas funções, apontadas por Reichert et al. (2003) como suporte para crescimento de plantas, regulagem de fluxos de energia e matéria do ambiente e filtro ambiental.

Solos cultivados, não saturados, são particularmente influenciados por problemas de compactação, que ocorre quando a pressão aplicada por rodados de máquinas e, ou, pisoteio animal ultrapassa a sua capacidade de suporte de carga, determinada pela pressão de préconsolidação $\left(\sigma_{p}\right)$, gerando deformações plásticas na sua estrutura, perdurando por anos. A magnitude dessas deformações depende de fatores extrínsecos, como tipo e massa das máquinas, tipo de rodados e pneus, carga sobre pneus e pressão de insuflagem, velocidade de deslocamento e tipo de cobertura vegetal (Zhao et al., 2010), e intrínsecos do solo, como densidade inicial, conteúdo de água, teor de argila, agregação e mineralogia (Ajayi et al., 2009) e matéria orgânica (Braida et al., 2008).
Os efeitos da compactação sobre as características e propriedades do solo evidenciam aumento na densidade, redução na porosidade total, bem como na infiltração e no armazenamento de água nele (Dias Junior \& Pierce, 1996) e restrição ao fluxo de gases e aumento na resistência ao crescimento de raízes (Hamza \& Anderson, 2005). Esses efeitos têm sua máxima expressão principalmente em períodos de déficit hídrico, ao passo que em períodos com normalidade da precipitação pluvial tais efeitos são, aparentemente, anulados.

No entanto, são escassos estudos que avaliam as respostas do uso de plantas e de sistema de rotação de culturas em plantio direto sobre a $\sigma_{p}$, bem como sobre a suscetibilidade do solo à compactação, indicada pelo índice de compressibilidade (IC). Sabese que as plantas modificam o ambiente do solo, melhorando a estrutura e aumentando a estabilidade dos agregados (Conceição et al., 2005), podendo refletir em aumento (Veiga et al., 2007) ou redução da capacidade de suporte de carga (Debiasi et al., 2008). Esses autores apontam que o uso de plantas de cobertura no inverno, aliado à ausência de tráfego, reduz a densidade do solo e aumenta a macroporosidade, diminuindo a capacidade de suporte de carga desse, bem como aumentando a 
suscetibilidade dele à compactação, limitando-se, porém, à camada superficial.

Embora Conceição et al. (2005) apontem que as plantas modificam o ambiente do solo, elas, por sua vez, são influenciadas pela compactação desse e disponibilidade hídrica, pois o nível de compactação (Souza et al., 2006) e o teor de umidade (Bengough et al., 2006) podem reduzir ou até impedir o crescimento radicular. A redução do conteúdo de água no solo aumenta a coesão entre partículas e a resistência à penetração (RP) e diminui a pressão hidrostática das células das raízes (Hamza \& Anderson, 2005). Em consequência, há redução da pressão exercida pela coifa e região meristemática, restringindo o crescimento radicular (Hamza \& Anderson, 2005) em valores de resistência à penetração acima de $2,0 \mathrm{MPa}$ (Silva et al., 1994).

Diante disso, o objetivo deste trabalho foi avaliar atributos físicos de solo e de planta, estas cultivadas em condições controladas. Acredita-se que: o uso de plantas de cobertura do solo no inverno associado à rotação de culturas no verão conferiu ao sistema plantio direto maior capacidade de suporte de carga e menor suscetibilidade à compactação; com a capacidade de suporte de carga, mesmo quando ultrapassada, o solo retorna à condição original em razão do sistema de manejo empregado; não havendo restrição hídrica, as plantas não teriam o crescimento e desenvolvimento radicular interferido pelo tráfego de máquinas.

Para testar essas hipóteses foram mensuradas propriedades de solo como a pressão de pré-consolidação, a resistência mecânica do solo à penetração, a densidade do solo, a porosidade total, o diâmetro médio ponderado de agregados, e parâmetros de planta como a área foliar, a massa da parte aérea e radicular de raízes de milho.

\section{MATERIAL E MÉTODOS}

O trabalho consta de dois estudos: um no campo com posterior análise do material em laboratório e outro em casa de vegetação, conduzidos em vasos. $\mathrm{O}$ experimento está localizado na Estação Experimental Agronômica da Universidade Federal do Rio Grande do Sul, no município de Eldorado do Sul, em Argissolo Vermelho distrófico (Embrapa, 2006). Parcelas (4,5 x $20 \mathrm{~m}$ ) estão sendo conduzidas em blocos ao acaso com três repetições desde 0 ano de 2002, em sistema plantio direto, com duas coberturas no inverno: aveia preta + ervilhaca e pousio, ambas cultivadas no verão com rotação de culturas (milho/soja), todas sob tráfego controlado de máquinas.

Desde a implantação do experimento (2002) até a safra de inverno de 2011, houve tráfego nas parcelas, duas vezes ao ano, por um trator John Deere, modelo 5600 , com massa de $3,8 \mathrm{t}$, com pneus dianteiros 12.4$24 \mathrm{R} 1$ e traseiros 18.4-30 R1, com pressão de insuflagem de 95 e $110 \mathrm{kPa}$, respectivamente. Na safra de verão 2012, foi utilizado um trator Valtra, modelo
BM125i, com massa de 5,3 t, com pneus dianteiros 14.9-26R1 e traseiros 23.1-30R1, com pressão de insuflagem de 219,78 e $153,18 \mathrm{kPa}$, respectivamente. A pressão de contato dos pneus dianteiros e traseiros com o solo, determinada pelo método de O'Sullivan et al. (1999) para o trator John Deere e Valtra, foi de 115 e $120 \mathrm{kPa}$ e 183 e $155 \mathrm{kPa}$, respectivamente.

Para o estudo no campo, amostras de solo indeformadas foram coletadas em cada parcela nos locais sem tráfego de trator (ST), antes do tráfego na safra 2012 (AT), ou seja, com acúmulo de pressões aplicadas nas safras anteriores com trator de menor massa (trator John Deere de 3,8 t), e depois do tráfego da safra 2012 (DT), quando da mudança do trator utilizado (trator Valtra BM $125 \mathrm{i}$ de 5,3 t), nas camadas de $0,0-0,05 ; 0,05-0,10 ; 0,10-0,15$; e 0,15-0,20 m. Em cada amostra dessas camadas foram determinadas a distribuição granulométrica (Quadro 1), a densidade do solo (Ds), a porosidade (PT), a pressão de préconsolidação $\left(\sigma_{\mathrm{p}}\right)$ e a distribuição e os agregados por classe de tamanho.

A resistência do solo à penetração $(\mathrm{RP})$ foi determinada em transecta com 15 pontos em cada parcela, utilizando um penetrômetro dotado de sistema eletrônico de medição e aquisição de dados de força aplicados à respectiva profundidade no solo (ASAE, 2004). Amostras indeformadas com volume de $77,90 \mathrm{~cm}^{3}(6,3$ e $2,5 \mathrm{~cm}$ - diâmetro e altura, respectivamente) de cada camada foram submetidas à tensão de $6 \mathrm{kPa}$ em mesa de tensão e, posteriormente, determinada a $\sigma_{\mathrm{p}}$ em uma prensa oedométrica, marca TESTOP, seguindo método descrito pela norma NBR 12007/90 (ABNT, 1990), com modificações sugeridas por Carpenedo (1994). Amostras deformadas, com estrutura preservada, constituída de agregados, foram coletadas naquelas camadas, para a determinação da estabilidade de agregados em água (EA), de acordo com Carpenedo \& Mielniczuk (1990). A Ds e a PT foram definidas, segundo método proposto por Embrapa (1997).

Quadro 1. Distribuição granulométrica das partículas do solo em diferentes camadas em razão das coberturas de inverno

\begin{tabular}{lcccc}
\hline $\begin{array}{l}\text { Cobertura de } \\
\text { inverno }\end{array}$ & Camada & Areia & Silte & Argila \\
\hline \multirow{2}{*}{ Pousio } & $\mathrm{m}$ & & $\mathrm{g} \mathrm{kg}^{-1}$ & \\
\cline { 3 - 5 } & $0,00-0,05$ & 538 & 217 & 245 \\
& $0,05-0,10$ & 534 & 198 & 268 \\
& $0,10-0,15$ & 521 & 186 & 293 \\
Aveia + ervilhaca & $0,15-0,20$ & 505 & 184 & 311 \\
& $0,00-0,05$ & 505 & 230 & 265 \\
& $0,05-0,10$ & 509 & 209 & 282 \\
& $0,10-0,15$ & 498 & 211 & 291 \\
& $0,15-0,20$ & 510 & 181 & 309 \\
\hline
\end{tabular}


Para o estudo desenvolvido em casa de vegetação, foram coletadas amostras indeformadas de solo na camada de 0,0-0,2 m (vasos de PVC com diâmetro de $0,1 \mathrm{~m}$ e altura de $0,2 \mathrm{~m}$ ) nas parcelas sob pousio, antes e depois do tráfego (AT e DT, respectivamente). Em cada parcela, coletaram-se oito amostras (quatro AT e quatro DT), totalizando 24 amostras. Em cada vaso, foram semeadas três sementes de milho cultivadas durante 25 dias. Das 24 amostras, seis AT e seis DT foram cultivadas sem restrição hídrica ( $\mathrm{SRH}$; média de 5,0 mm diários de lamina de água), e as outras seis AT e seis DT com restrição hídrica $(\mathrm{CRH}$; média de 3,5 mm diários de lamina de água). Ao final do período de condução, determinaram-se a área foliar, massa verde e seca da parte aérea e massa úmida e seca das raízes. $\mathrm{O}$ volume de solo do cilindro foi disposto sobre placas de pregos, e as raízes lavadas com água e posterior estratificação em camadas de $0,05 \mathrm{~m}$, até $0,2 \mathrm{~m}$.

Os dados foram submetidos à análise estatística pelo software Statistycal Analysis System versão 9.0 (SAS for Windows) e, havendo significância na análise de variância, foram comparadas as médias pelo teste de Tukey $(\mathrm{p}<0,05)$.

\section{RESULTADOS E DISCUSSÃO}

\section{Propriedades físicas do solo influenciadas pela cobertura de inverno e pelo tráfego}

A densidade do solo (Ds), independentemente da profundidade, não foi alterada pelas condições de manejo. No pousio, não foram observadas diferenças nos valores de Ds entre as condições de tráfego em cada camada, mas sim entre camadas em cada condição de tráfego até $0,10 \mathrm{~m}$ (Figura 1a). No consórcio aveia + ervilhaca, foram observadas diferenças entre condições de tráfego e profundidade para o ST até 0,10 $\mathrm{m}$ e, para o DT, até $0,05 \mathrm{~m}$, não havendo diferenças em profundidade para o AT. Essa dinâmica provavelmente deve-se ao teor de matéria orgânica no solo na camada até $0,13 \mathrm{~m}$ para pousio e aveia + ervilhaca, 63,77 e $68,43 \mathrm{~g} \mathrm{~kg}^{-1}$, respectivamente, avaliada por Sequinatto (2010), e ao incremento de argila em profundidade (Quadro 1), característica dos Argissolos, que deposita-se entre os grãos de areia, aumentando a massa do solo em mesmo volume.

Os valores de porosidade (macro e micro) não diferiram entre as condições de manejo (pousio e aveia + ervilhaca), em cada condição de tráfego e profundidade (dados não apresentados). No entanto, em valores absolutos, a macroporosidade apresentou tendência de diminuição em profundidade. No pousio, na camada $0,05 \mathrm{~m}$, houve diferença na macroporosidade entre a condição AT e DT, enquanto para a aveia + ervilhaca houve diferença entre a condição ST e DT (Figura 2a,b). Em ambas as condições de manejo, os menores valores ocorreram em DT. Esse comportamento está associado à Ds que, embora não diferindo na condição de pousio, apresentou, em valores absolutos, tendência de aumento em DT. Além disso, observou-se aumento de "agregados" (denominação em razão do método usado, porém, nesse caso, a denominação blocos a agregados é melhor representada) da classe $\geq 2,00 \mathrm{~mm}$, após o tráfego, indicando que essa classe de "agregados" foi formada mecanicamente, por pressão externa (Figura 3), o que diminuiu a quantidade de macroporos; entretanto, interferindo pouco a densidade total na camada avaliada. Essa hipótese condiz com o estudo realizado por Carrasco (1989), o qual analisando a estrutura do solo em micro escala, revelou redução em tamanho e número de macroporos, bem como mudanças na sua forma e continuidade.

Em profundidade, no pousio (Figura 2a), a macroporosidade diferiu nas condições de tráfego AT, cujo limite foi a camada de 0,10 m, e DT, de 0,05 m. Para a condição ST, no pousio, e para todas as condições ST, AT e DT, na aveia + ervilhaca, não foram observadas diferenças nessa propriedade (Figura 2a,b). Isso se deve à Ds que apresentou comportamento inverso à porosidade, bem como ao incremento de argila em profundidade, evidenciado em ambas as condições de manejo (Quadro 1).

Os valores de microporosidade apresentaram diferença entre as condições de tráfego AT e DT somente na camada até $0,05 \mathrm{~m}$ para pousio. Nas demais camadas, para o pousio, e em todas as camadas na condição de manejo aveia + ervilhaca, não foram observadas diferenças entre as condições de tráfego (Figura 2a,b).

Em profundidade, a microporosidade diferiu nas condições de tráfego ST e AT, no pousio, com menores valores na camada até $0,10 \mathrm{~m}$ (Figura $2 \mathrm{a}$ ). Na condição DT, no pousio, e em todas as condições de tráfego na aveia + ervilhaca, não foi observada diferença significativa nessa característica (Figura 2a,b). Essa característica dificilmente é influenciada pelo manejo, uma vez que a microporosidade está relacionada com a organização das partículas minerais primárias do solo isoladas e, ou, em microagregados (Lal \& Shukla, 2005).

A pressão de pré-consolidação, independentemente da condição de tráfego, apresentou maiores valores absolutos no manejo aveia + ervilhaca (Quadro 2). Para a condição ST (Quadro 2), houve diferença entre os sistemas de manejo na camada $0,15-0,20 \mathrm{~m}$; na de tráfego AT, nas camadas 0,05-0,10 e 0,15-0,20 m; e para a condição DT, na camada $0,10-0,15 \mathrm{~m}$. Atribuiuse a isso a dois fatores: o primeiro, evidenciou-se que mantendo o solo permanentemente coberto com plantas que aportam grande quantidade de matéria e energia (ex. gramíneas + leguminosas), aumenta-se a entropia do sistema, o que leva a novo estado de ordem, aumentando a resistência a perturbações e resiliência do sistema solo (Vezzani \& Mielniczuk, 2011), o que melhora, por exemplo, propriedades como a porosidade, evidenciadas na figura 2; e o segundo está associado 


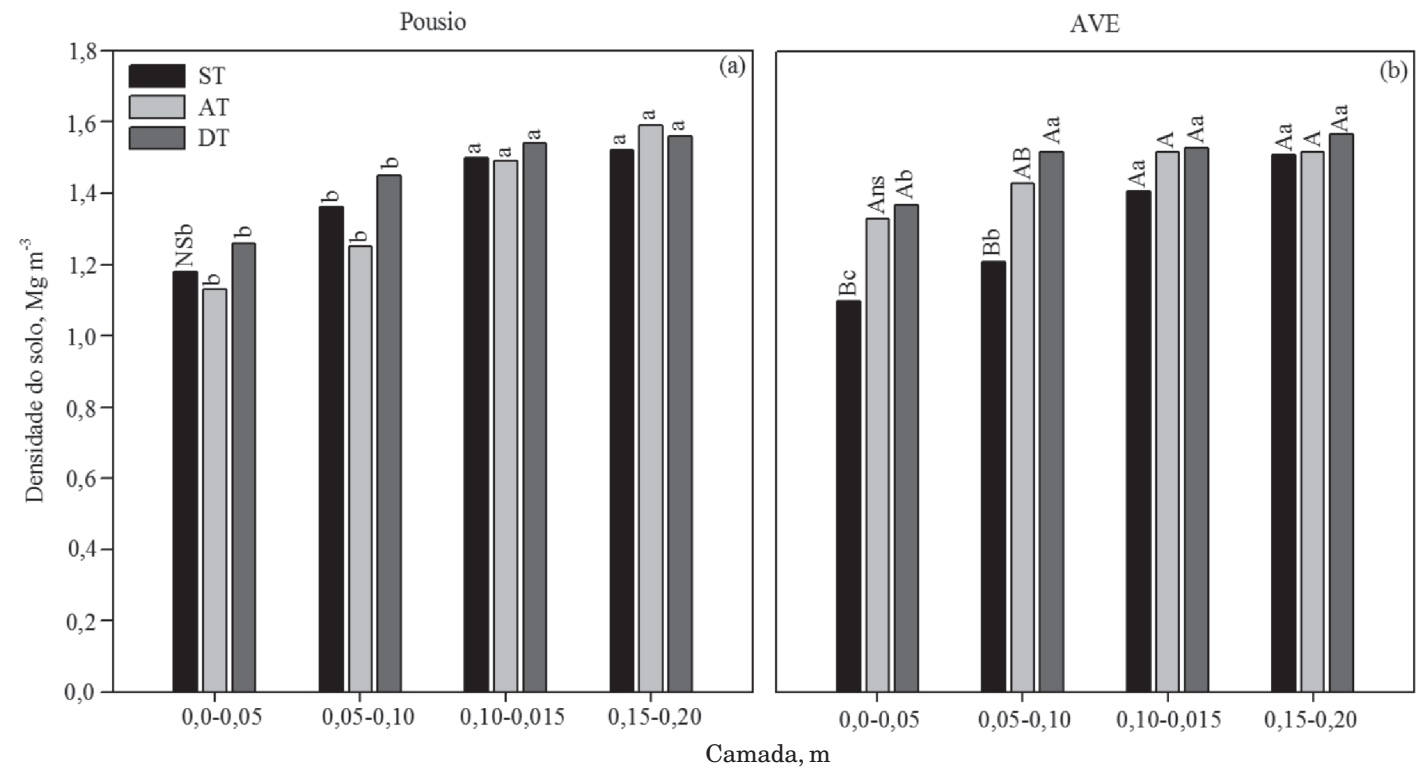

Figura 1. Densidade do solo nos sistemas de manejo pousio (a) e aveia + ervilhaca (b), nas condições de sem tráfego (ST), antes do tráfego (AT) e depois do tráfego (DT), em camadas. Letras iguais não diferem significativamente pelo teste de Tukey $(p<0,05)$. Letras maiúsculas comparam condições de tráfego em cada camada e minúsculas, camadas em cada condição de tráfego.
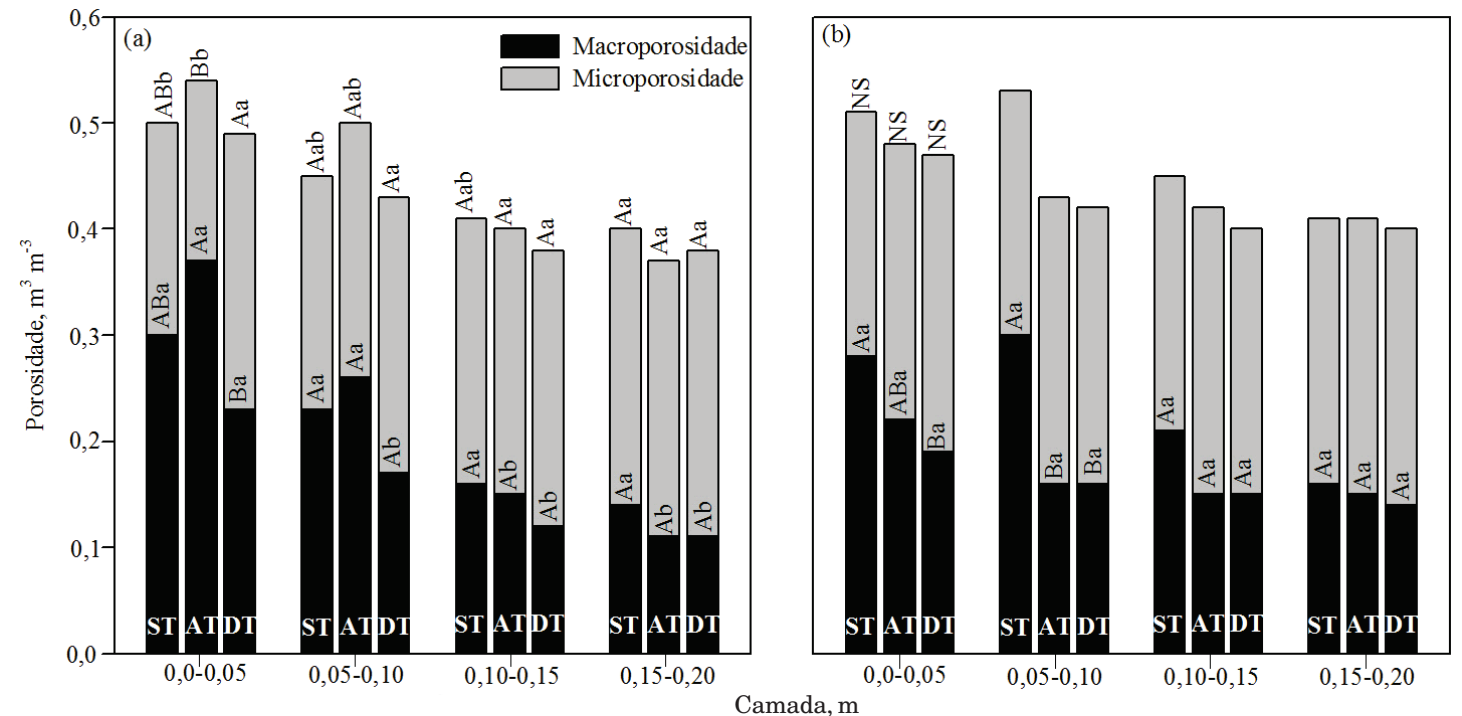

Figura 2. Porosidade do solo nos sistemas de manejo pousio (a) e aveia mais ervilhaca (b), nas condições de sem tráfego (ST), antes do tráfego (AT) e depois do tráfego (DT), em camadas. Letras iguais não diferem significativamente pelo teste de Tukey $(p<0,05)$. Letras maiúsculas comparam condições de tráfego em cada camada e minúsculas, camadas em cada condição de tráfego.

ao efeito do histórico de tráfego na área, ou seja, a intensidade de tráfego imposta pelo trator utilizado na área experimental até 2011 tem aumentado a Ds (Figura 2), contribuindo para a diminuição do índice de compressibilidade, evidenciado pela correlação negativa no quadro 4, e aumento na pressão de préconsolidação (Schäffer et al., 2007).

No pousio, houve diferença na pressão de préconsolidação entre condições de tráfego nas camadas
0,0-0,05 e 0,15-0,20 m, maiores valores observados para a condição DT. Entre camadas, ocorreu diferença para as condições ST e DT, sendo os maiores valores observados na camada 0,15-0,20 m (Figura 4a). Isso pode ser explicado pela menor macroporosidade e maior DS da camada superficial, especialmente na condição DT, ocasionada pelo tráfego e pelo incremento natural de argila em profundidade, acarretando aumento na Ds (Figura 1a), ou seja, há um incremento de massa 
em mesmo volume. Embora essa não tenha apresentado diferença entre as condições de tráfego, a transmissão de pressão e a vibração imprimida no solo pelos pneus acarretaram aumento da quantidade de "agregados" da classe $\geq 2,00 \mathrm{~mm}$ (Figura 3), sugerindo ser uma junção mecânica das partículas de solo oriundas do tráfego. Logo, pressões externas levam a novo rearranjo desses agregados, reduzindo a macroporosidade (Carrasco, 1989); entretanto, sem efeitos sobre a Ds neste estudo.

Para a aveia + ervilhaca, houve diferença na pressão de pré-consolidação entre condições de tráfego somente na camada $0,15-0,20 \mathrm{~m}$, com maiores valores nos tratamentos em que ocorreu tráfego no passado (Figura 4b). Isso provavelmente deve-se ao fato de que, abaixo de $0,15 \mathrm{~m}$, há pouca contribuição dos resíduos vegetais no processo de agregação, ficando esse processo predominantemente por ação de ligações entre argilominerais e óxidos (Lal \& Shukla, 2005) ou mesmo de forças mecânicas externas, como histórico de pressões aplicadas pelo tráfego.

Comparando camadas em cada condição de tráfego, verificou-se menor valor da pressão de pré-consolidação na camada 0,05 m (ST e AT), essa diferindo das camadas abaixo de $0,10 \mathrm{~m}$ na condição ST, e para todas as camadas na condição AT. Para a condição DT, os menores valores foram encontrados nas camadas até $0,10 \mathrm{~m}$, essas diferindo das demais, provavelmente em razão do aumento no teor de argila em profundidade (Quadro 1), ocasionando aumento natural nos valores de Ds (Figura 1) e no acúmulo de pressões pelo tráfego.

A resistência do solo à penetração $(\mathrm{RP})$ apresentou comportamento semelhante às demais análises, aumentando em profundidade para ambas as condições
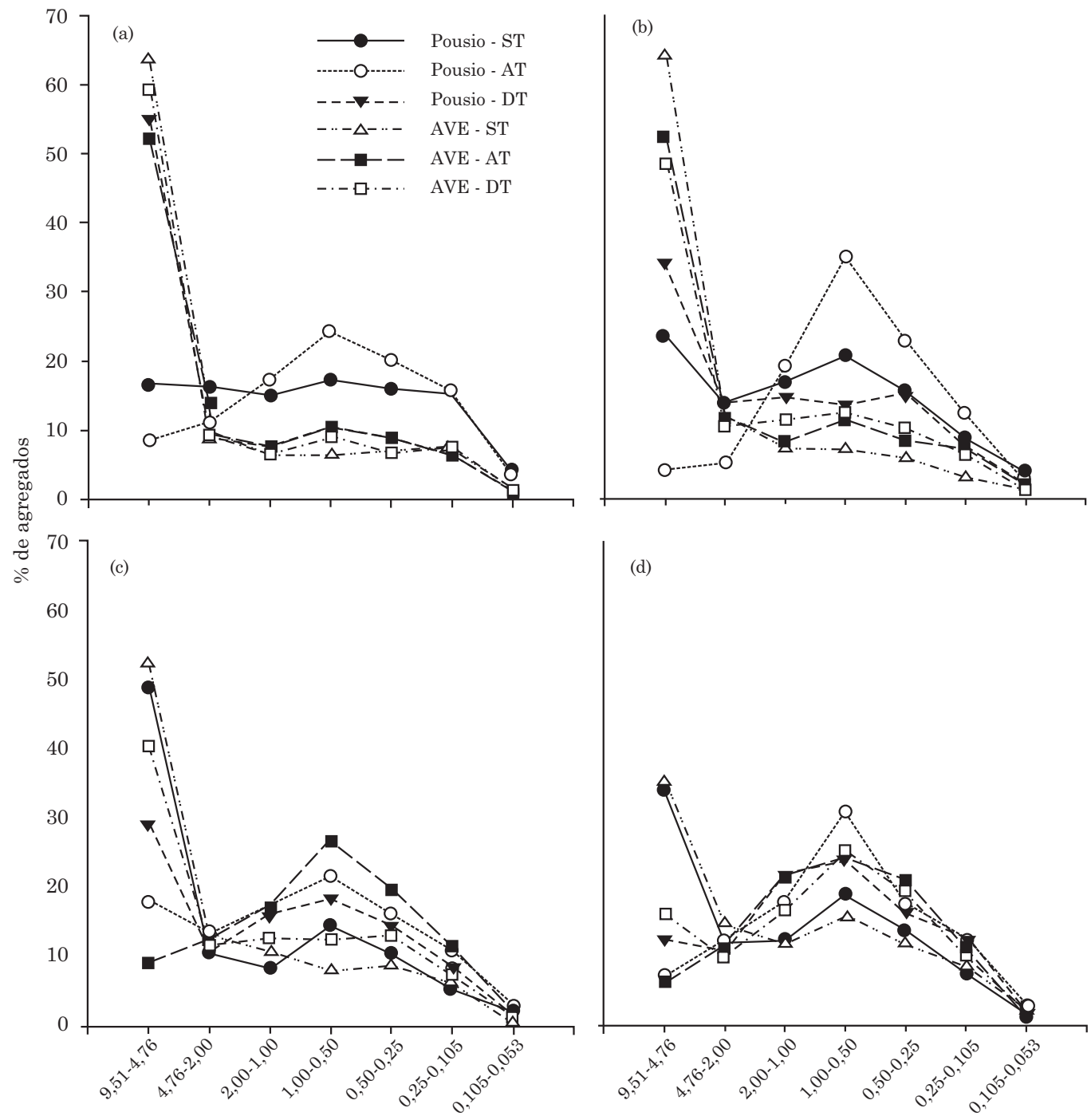

Classe de diâmetro, $\mathrm{mm}$

Figura 3. Distribuição de agregados por classes de tamanhos nos sistemas de manejo e nas condições de tráfego, nas profundidades 0,0-0,05 (a), 0,05-0,10 (b), 0,10-0,15 (c) e 0,15-0,20 m (d). AVE: aveia + ervilhaca; ST: sem tráfego; AT: antes do tráfego; e DT: depois do tráfego. 
de manejo (Figura 5), porém com magnitude dos valores distintos. Para a cobertura de inverno pousio (Figura 5a), os valores de RP variaram de 0,4 a 1,6 $\mathrm{MPa}$, apresentando pequena amplitude entre as condições de tráfego, em razão da pequena variação na Ds (Figura 1a) e profundidade (até $0,10 \mathrm{~m}$ ).

Para a cobertura aveia + ervilhaca, a magnitude na variação dos valores foi maior $(0,4 \mathrm{a} 2,1 \mathrm{MPa})$, com valores de RP próximos entre as condições de tráfego AT e DT (Figura 5b) até a profundidade 0,15 $\mathrm{m}$. Tendo como referência os valores de RP da condição ST, observou-se que as condições de tráfego na cobertura aveia + ervilhaca impostas ao solo com o trator até 2011 (condição AT) aumentou a RP até

Quadro 2. Pressão de pré-consolidação comparando sistemas de manejo em cada camada e tráfego

\begin{tabular}{ccccc}
\hline \multirow{2}{*}{ Camada } & Manejo & \multicolumn{3}{c}{ Tráfego } \\
\cline { 3 - 5 } & & ST & AT & DT \\
\cline { 3 - 5 } $\mathrm{m}$ & & $\mathrm{kPa}$ & \\
$0,0-0,05$ & Pousio & $30 \mathrm{a}$ & $48 \mathrm{a}$ & $54 \mathrm{a}$ \\
& AVE & $40 \mathrm{a}$ & $47 \mathrm{a}$ & $56 \mathrm{a}$ \\
$0,05-0,10$ & Pousio & $43 \mathrm{a}$ & $53 \mathrm{~b}$ & $75 \mathrm{a}$ \\
& AVE & $60 \mathrm{a}$ & $90 \mathrm{a}$ & $76 \mathrm{a}$ \\
$0,10-0,15$ & Pousio & $54 \mathrm{a}$ & $80 \mathrm{a}$ & $76 \mathrm{~b}$ \\
& AVE & $98 \mathrm{a}$ & $105 \mathrm{a}$ & $128 \mathrm{a}$ \\
$0,15-0,20$ & Pousio & $61 \mathrm{~b}$ & $69 \mathrm{~b}$ & $129 \mathrm{a}$ \\
& AVE & $85 \mathrm{a}$ & $121 \mathrm{a}$ & $132 \mathrm{a}$ \\
\hline
\end{tabular}

ST: sem tráfego; AT: antes do tráfego; DT: depois do tráfego; AVE: aveia + ervilhaca. Letras iguais na coluna comparam sistemas de manejo em mesma camada e tráfego, não diferindo significativamente pelo teste de Tukey a $5 \%$.
$0,15 \mathrm{~m}$ de profundidade (Figura $5 \mathrm{~b}$ ). Um novo acréscimo de carga (imposto pelo sistema DT) promoveu incremento dos valores de RP e Ds até $0,20 \mathrm{~m}$ de profundidade, atingindo valor de 2,1 $\mathrm{MPa} \mathrm{e}$ $1,57 \mathrm{Mg} \mathrm{m}^{-3}$, respectivamente. Esses dados corroboram com Imhoff (2002), o qual apontou que valores de RP maiores que 2,0 $\mathrm{MPa}$ limitariam o crescimento radicular, bem como com Reichert et al. (2003), os quais afirmaram que a densidade crítica para desenvolvimento radicular para esse solo é de $1,5 \mathrm{Mg} \mathrm{m}^{-3}$, o que nesse caso pode vir a acontecer. Isso sugere que a frequência de tráfego ao longo dos anos pode ocasionar problemas de compactação em subsuperfície.

Comparando as coberturas de inverno, com base nos valores de RP (Figura 5a,b), a maior magnitude dos valores poderia ter ocorrido em razão da mudança no conteúdo gravimétrico de água no solo no momento das avaliações. Tal fato não ocorreu, uma vez que os valores de umidade foram de $0,15 \pm 0,01$ e $0,17 \pm 0,01 \mathrm{~kg} \mathrm{~kg}^{-1}$ para pousio e aveia + ervilhaca, respectivamente, valores esses abaixo do limite de plasticidade $\left(0,18 \mathrm{~kg} \mathrm{~kg}^{-1}\right)$, determinado por Mazurana (2011) para mesmo solo. Dessa forma, a maior magnitude nos valores de RP observada entre as duas condições de cobertura pode ser resultado de maior estabilidade na estrutura do solo no sistema aveia + ervilhaca, uma vez que o seu teor de matéria orgânica desse sistema é maior que o pousio (Sequinatto, 2010). No entanto, esse aumento na RP não é entendido como impedimento ao crescimento radicular, mas à maior estabilidade da estrutura do solo a deformações.

\section{Características de planta influenciadas pelo tráfego e pela disponibilidade hídrica}

As características de planta avaliadas em casa de vegetação nas condições de disponibilidade hídrica e

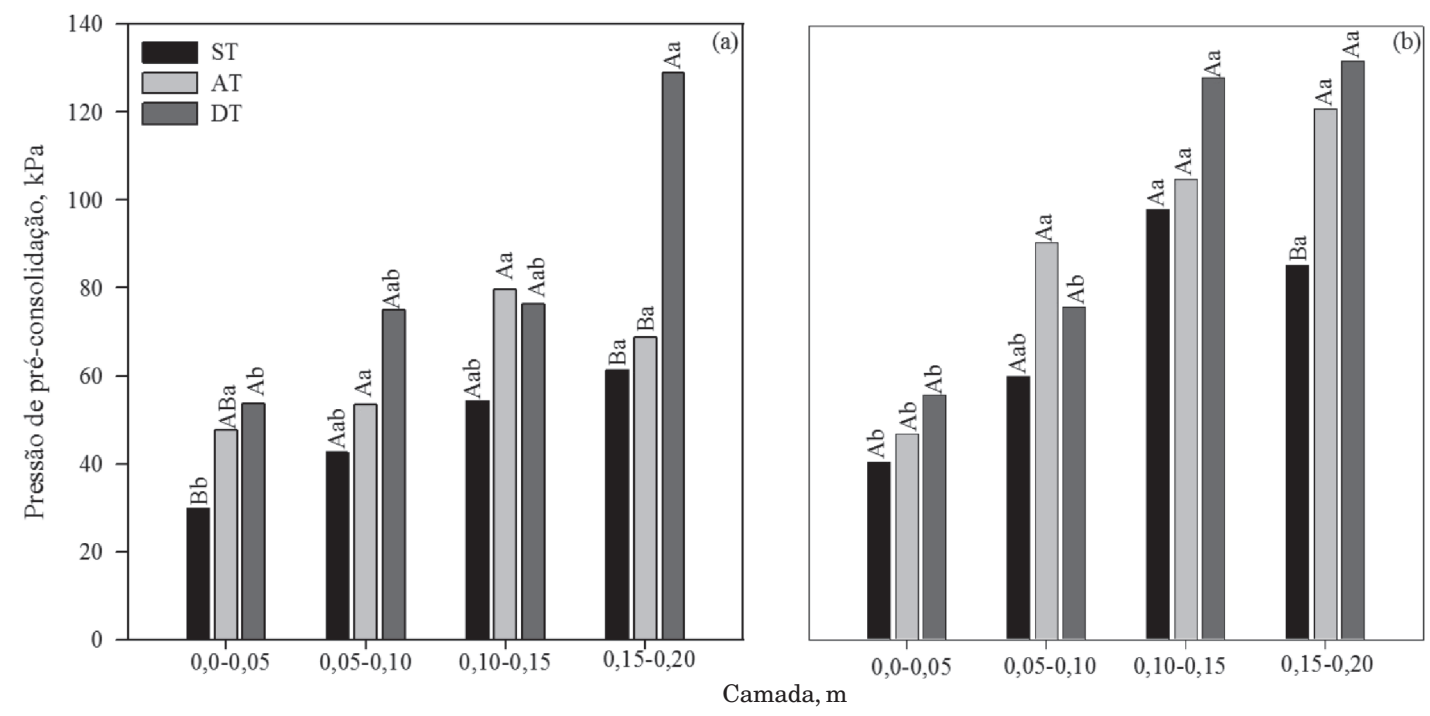

Figura 4. Pressão de pré-consolidação nos sistemas de manejo pousio (a) e aveia + ervilhaca (b), nas condições de sem tráfego (ST), antes do tráfego (AT) e depois do tráfego (DT), em camadas. Letras iguais não diferem significativamente pelo teste de Tukey $(p<0,05)$. Letras maiúsculas comparam condições de tráfego em cada camada e minúsculas, camadas em cada condição de tráfego. 


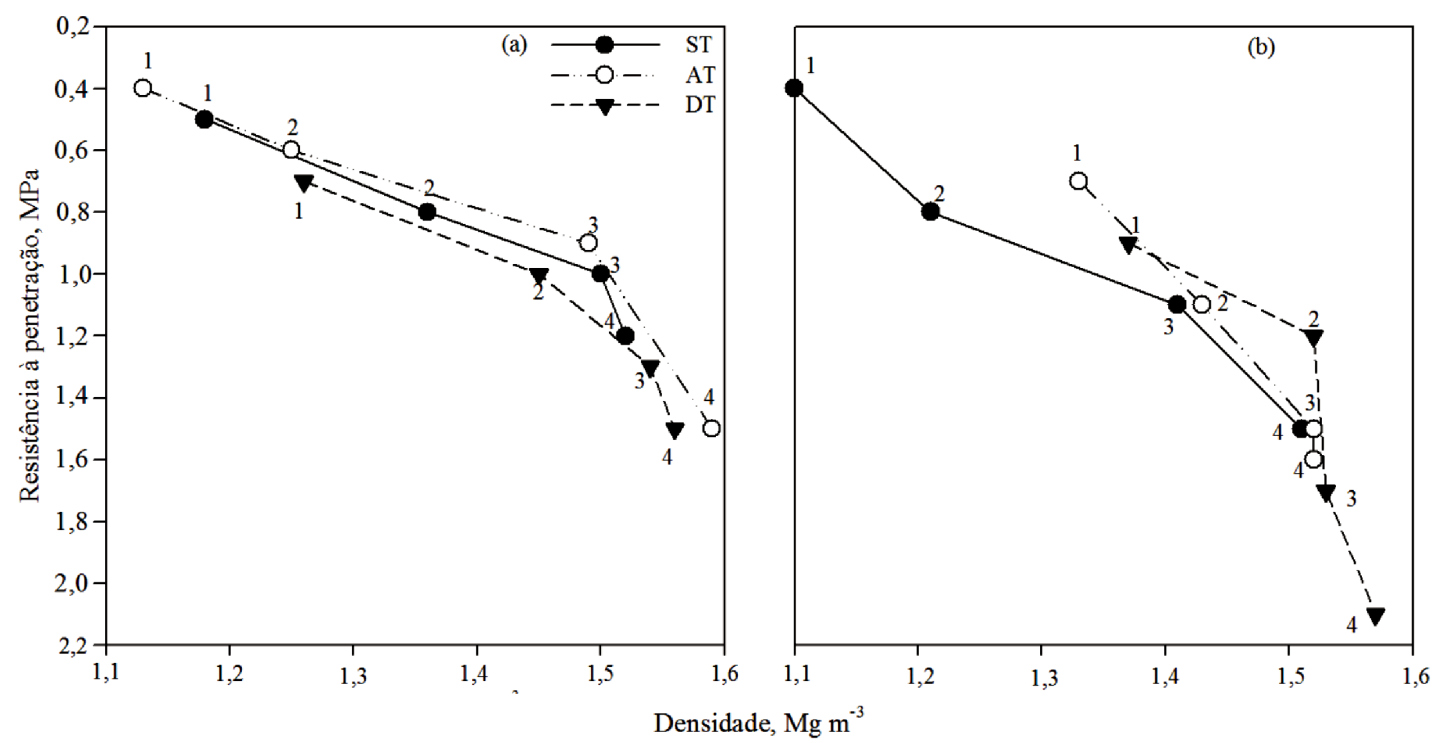

Figura 5. Resistência mecânica do solo à penetração e densidade no solo na condição de pousio (a) e aveia + ervilhaca (b). Números sobre os marcadores indicam camadas no solo 0,0-0,05 (1), 0,05-0,10 (2), 0,10-0,15 (3) e 0,15-0,20 m (4).

tráfego controlado estão apresentados no quadro $3 . \mathrm{O}$ tratamento com restrição hídrica $(\mathrm{CRH})$ apresentou menor produção de massa de raízes do milho, em relação ao tratamento sem restrição hídrica (SRH). Possivelmente, no tratamento com $\mathrm{CRH}$, o maior potencial matricial de água no solo, em razão da drenagem natural e, ou, evapotranspiração, aumentou a coesão entre partículas desse, elevando a resistência ao crescimento da raiz. Além disso, menor quantidade de água chega e ascende às células das raízes, diminuindo a pressão hidrostática e não mantendo o mínimo de turgor necessário para a expansão celular (Hsiao \& Xu, 2000). Ambos os fatores associados diminuem ou até mesmo impedem o crescimento radicular, como apontam Hamza \& Anderson (2005).

A produção de raízes diminuiu em profundidade em todos os tratamentos (Quadro 3). No tratamento $\mathrm{CRH}$, somente a primeira camada diferiu das demais, enquanto as camadas inferiores apresentaram tendência na diminuição dos valores absolutos. Para o tratamento SRH, houve diferenças entre as camadas, com maiores valores para a camada até $0,10 \mathrm{~m}$. No entanto, a camada $0,10-0,15$ m não diferiu das de 0,05 0,10 e $0,15-0,20 \mathrm{~m}$, indicando estagnação no crescimento das raízes. Segundo Barber et al. (1988), a taxa de crescimento das raízes de milho é altamente dependente do conteúdo de água no solo; a maior disponibilidade auxilia na turgidez das células radiculares, aumentando a expansão celular, bem como atua como lubrificante entre a coifa e as partículas de solo, permitindo a melhor penetração das raízes em crescimento.

Analisando os resultados para uma mesma camada, observou-se que não há diferença entre os tratamentos nas camadas $0,0-0,05$ e $0,15-0,20 \mathrm{~m}$. No entanto, nas duas camadas intermediárias, os maiores valores de massa de raízes foram observados no tratamento DT-SRH ( 1,26 e $0,77 \mathrm{~g})$, não diferindo do tratamento AT-SRH $(0,80$ e 0,54 g, respectivamente). Esses resultados sugeriram que o tratamento DT-SRH proporcionou melhores condições comparativamente ao tratamento AT-SRH para o desenvolvimento das raízes (Quadro 3), fato atribuído à maior aproximação das partículas de solo às raízes, aumentando o volume de solo explorado. Isso corrobora com Barber (1995), o qual, avaliando quatro níveis de compactação, verificou aumento de absorção de nutrientes pelas plantas na medida em que aumentou o nível de compactação, principalmente pela diminuição da tortuosidade do caminho difusivo para fósforo e potássio e aproximação das partículas do solo para o fluxo de massa e intercepção radicular para cálcio e magnésio, evidenciando ter a compactação, em certo grau, efeito benéfico no desenvolvimento das plantas.

Em relação à produção de matéria da parte aérea e à área foliar, somente houve diferença entre as médias dos tratamentos SRH e $\mathrm{CRH}$, sem haver influência do tráfego (Quadro 3). No entanto, a relação entre parte aérea e raízes foi menor nos tratamentos $\mathrm{SRH}$, apresentando que a maior disponibilidade de água no solo promoveu o maior crescimento das raízes em razão da menor resistência do solo à penetração (Libardi \& Jong van Lier, 1999), entendido como efeito lubrificante entre raiz-solo e diminuição nas forças de coesão que mantêm unidas as partículas na estrutura do solo. Nesse mesmo tratamento, a umidade das raízes apresentou tendência em ser menor que no tratamento $\mathrm{CRH}$. Isto pode ser indicativo de que, em déficit hídrico, a planta acumula mais solutos em suas células, retendo mais água. Ainda, segundo Hsiao \& $\mathrm{Xu}$ (2000), as plantas têm alta capacidade de rápido ajuste osmótico do potencial de água das células das 
raízes, o que não ocorre nas folhas. Desse modo, para baixo potencial de água no solo, as folhas tendem a diminuir a taxa de crescimento antes que as raízes.

\section{Correlações entre características avaliadas}

No quadro 4, são apresentados os coeficientes de correlação de Pearson e seus respectivos níveis de significância. A Ds evidenciou relação negativa com a macroporosidade, a porosidade total e o IC. Esse resultado deve-se ao fato de que a diminuição do espaço aéreo do solo em razão de tráfego ou manejo aumentou a densidade dele, embora não evidenciado neste trabalho, o que, por sua vez, diminuiu a capacidade desse em se deformar (IC), aumentando a sua capacidade em suportar carga $\left(\sigma_{p}\right)$.

Os sistemas de manejo e tráfego influenciaram a macroporosidade (Figura 2) e isso refletiu diretamente na porosidade total, explicando a relação direta entre esses dois parâmetros ( $\mathrm{r}=0,9365 ; \alpha<0,0001)$ (Quadro 4). A correlação entre macro e microporosidade $(\mathrm{r}=0,7156$; $\alpha<0,0001$ ), não é um efeito direto (Quadro 4), corroborando com o fato de que a microporosidade é pouco influenciada pelas cobertura de inverno e tráfego, mas sim por características intrínsecas de solo. No entanto, a proporção relativa de microporos, em relação aos macroporos, aumentou quando a macroporosidade diminuiu por efeitos de manejo, por exemplo.

A relação entre a produção de raízes de milho foi negativa com a Ds $(r=-0,7640 ; \alpha<0,0001)$ e positiva com a macroporosidade $(r=0,6874 ; \alpha=0,0002)$ (Quadro 4). Possivelmente, essa dinâmica envolveu o aumento natural da Ds em profundidade (Figura 1a,b), associado ao efeito do tráfego em diminuir a macroporosidade em profundidade e ao padrão natural de desenvolvimento de raízes, uma vez que, encontrando condições adequadas de nutrição, disponibilidade hídrica e oxigenação nas camadas superficiais, não há dreno de energia para crescimento radicular em camadas profundas $(0,15-0,20 \mathrm{~m})$.

Quadro 3. Produção de raízes das camadas estratificadas, parte aérea (PA), área foliar (AF), relação entre parte aérea/raízes do milho (PA/R) e teor de umidade das raízes (Ura) para os tratamentos de restrição hídrica (CRH e SRH) e tráfego controlado (AT e DT)

\begin{tabular}{|c|c|c|c|c|c|c|c|c|c|}
\hline \multirow{2}{*}{ Tratamento } & \multicolumn{5}{|c|}{ Estratificação de raízes (m) } & \multirow{2}{*}{ PA } & \multirow{2}{*}{$\mathbf{A F}$} & \multirow{2}{*}{$\mathbf{P A} / \mathbf{R}$} & \multirow{2}{*}{ URa } \\
\hline & $0-0,05$ & 0,05-0,10 & $0,10-0,15$ & $0,15-0,20$ & $0-0,20$ & & & & \\
\hline & & & $\mathrm{g}(\mathrm{mas}$ & seca) & & & $\mathrm{cm}^{2}$ & & $\%$ \\
\hline $\mathrm{AT}-\mathrm{CRH}$ & $1,24{ }^{\mathrm{ns}} \mathrm{A}$ & $0,48 \mathrm{bB}$ & $0,23 \mathrm{cB}$ & $0,11^{\mathrm{ns}} B$ & $2,05 \mathrm{c}$ & $0,76 \mathrm{~b}$ & $148,2 \mathrm{~b}$ & 0,38 & $86^{\mathrm{ns}}$ \\
\hline DT - CRH & $1,36 \mathrm{~A}$ & $0,41 \mathrm{bB}$ & $0,31 \mathrm{bcB}$ & $0,09 \mathrm{~B}$ & $2,16 \mathrm{c}$ & $0,76 \mathrm{~b}$ & $137,6 \mathrm{~b}$ & 0,37 & 85 \\
\hline $\mathrm{AT}-\mathrm{SRH}$ & $1,59 \mathrm{~A}$ & $0,80 \mathrm{abB}$ & $0,54 \mathrm{abBC}$ & $0,44 \mathrm{C}$ & $3,37 \mathrm{~b}$ & $1,13 \mathrm{a}$ & $259,4 \mathrm{a}$ & 0,34 & 84 \\
\hline DT - SRH & $1,93 \mathrm{~A}$ & $1,26 \mathrm{aB}$ & $0,77 \mathrm{aBC}$ & $0,46 \mathrm{C}$ & $4,42 \mathrm{a}$ & $1,45 \mathrm{a}$ & 270,6 a & 0,33 & 84 \\
\hline
\end{tabular}

Letras maiúsculas comparam médias na linha e minúsculas, médias em colunas. ${ }^{\text {ns }}$ não significativo pelo teste de Tukey (p<0,05).

Quadro 4. Coeficientes de correlação de Pearson e nível de significância entre propriedades avaliadas

\begin{tabular}{|c|c|c|c|c|c|c|c|}
\hline & Ma & Mi & PT & DMP & $\sigma_{p}$ & IC & MR \\
\hline \multirow[t]{2}{*}{ DS } & $-0,9163$ & 0,5036 & $-0,9345$ & $-0,2731$ & 0,5726 & $-0,5726$ & $-0,7640$ \\
\hline & $<0,0001$ & $<0,0001$ & $<0,0001$ & 0,0203 & $<0,0001$ & $<0,0001$ & $<0,0001$ \\
\hline \multirow[t]{2}{*}{ Macro } & 1,0000 & $-0,7156$ & 0,9365 & 0,1977 & $-0,5177$ & 0,5462 & 0,6874 \\
\hline & & $<0,0001$ & $<0,0001$ & 0,0959 & $<0,0001$ & $<0,0001$ & 0,0002 \\
\hline \multirow[t]{2}{*}{ Micro } & & 1,000 & 0,4253 & 0,0477 & 0,2705 & $-0,3355$ & $-0,3023$ \\
\hline & & & 0,0002 & 0,6967 & 0,0216 & 0,0040 & 0,1510 \\
\hline \multirow[t]{2}{*}{ PT } & & & 1,0000 & 0,2802 & $-0,5350$ & 0,5393 & 0,7808 \\
\hline & & & & 0,0171 & $<0,0001$ & $<0,0001$ & $<0,0001$ \\
\hline \multirow[t]{2}{*}{ DMP } & & & & 1,0000 & $-0,2002$ & 0,0711 & 0,2990 \\
\hline & & & & & 0,0918 & 0,5525 & 0,1558 \\
\hline \multirow[t]{2}{*}{$\sigma_{\mathrm{p}}$} & & & & & 1,0000 & $-0,6755$ & $-0,5513$ \\
\hline & & & & & & $<0,0001$ & 0,0052 \\
\hline \multirow[t]{2}{*}{ IC } & & & & & & 1,0000 & 0,5572 \\
\hline & & & & & & & 0,0047 \\
\hline
\end{tabular}

Ma: macroporosidade; Mi: microporosidade; DS: densidade do solo; PT: porosidade total; $\sigma_{\mathrm{p}}$ : pressão de pré-consolidação; DMP: diâmetro médio ponderado; IC: índice de compressibilidade; e MR: massa de raiz. 


\section{CONCLUSÕES}

1. A densidade do solo, independentemente da camada avaliada, não foi influenciada pela condição de cobertura desse, mas sim pela condição de tráfego. Já a macroporosidade sofreu interferência do tráfego no sistema pousio até $0,10 \mathrm{~m}$, indicando ter esse sistema menor capacidade de suportar perturbações, comprovado também pelos menores valores de pressão de pré-consolidação.

2. A resistência do solo à penetração aumentou em profundidade, estando relacionada à maior densidade, menor macroporosidade e maior pressão de préconsolidação. Sem restrição hídrica, o crescimento radicular do milho foi influenciado positivamente pelo tráfego de máquinas. $\mathrm{O}$ tratamento $\mathrm{DT}$ sem restrição hídrica foi o que apresentou os valores mais altos de massa de raízes de milho, contrastando com o tratamento AT com restrição hídrica.

3. A produção de parte aérea e a área foliar não foram influenciadas pelas condições de tráfego. Nas condições da experimentação, o plantio direto com sistema de rotação de culturas evidenciou maior resiliência frente às perturbações do meio.

\section{AGRADECIMENTOS}

Aos professores Ibanor Anghinoni e Egon J. Meurer, pela dedicação, pelo ensinamento e incentivo antes, durante e após a realização deste trabalho.

\section{LITERATURA CITADA}

ASSOCIAÇÃO BRASILEIRA DE NORMAS TÉCNICAS ABNT. Ensaio de adensamento unidimensional: NBR 12007. Rio de Janeiro, 1990. 13p.

AJAYI, A.E.; DIAS JUNIOR, M.S.; CURI, N.; GONTIJO, I.; ARAUJO-JUNIOR, C.F. \& INDA JÚNIOR, A.V. Relation of strength and mineralogical attributes in Brazilian Latosols. Soil Till. Res., 102:14-18, 2009.

AMERICAN SOCIETY OF AGRICULTURAL ENGINEERS ASAE. Soil cone penetrometer - ASAE S313.3. Saint Joseph, 2004.

BARBER, S.A. Soil nutrient bioavailability: A mechanistic approach. 2.ed. New York, John Wiley \& Sons, 1995. $414 \mathrm{p}$.

BARBER, S.A.; MACKEY, A.D.; KUCHENBUCH, R.O. \& BARRACLOUGH, S. Effect of soil temperature and water on maize root growth. Plant Soil, 111:267-269, 1988.

BRAIDA, J.A.; REICHERT, J.M.; REINERT, D.J. \& SEQUINATTO, L. Elasticidade do solo em função da umidade e do teor de carbono orgânico. R. Bras. Ci. Solo, 32:477-485, 2008.
BENGOUGH, A.G.; BRANSBY, M.F.; HANS, J.; MCKENNA, S.J.; ROBERTS, T.J. \& VALENTINE, T.A. Root responses to soil physical conditions: growth dynamics from field to cell. J. Exp. Bot., 57:437-477, 2006.

CARPENEDO, V. Compressibilidade de solos em sistemas de manejo. Porto Alegre, Universidade Federal do Rio Grande do Sul, 1994. 106p. (Tese de Doutorado)

CARPENEDO, V. \& MIELNICZUK, J. Estado de agregação e qualidade de agregados de Latossolos Roxos, submetidos a diferentes sistemas de manejo. R. Bras. Ci. Solo, 14:99105, 1990.

CARRASCO, P.J. Efeito da compactação sobre as propriedades físicas do solo, crescimento e rendimento do girassol. Porto Alegre, Universidade Federal do Rio Grande do Sul, 1989. 107p. (Dissertação de Mestrado)

CONCEIÇÃO, P.C.; AMADO, T.J.C.; MIELNICZUK, J. \& SPAGNOLLO, E. Qualidade do solo em sistemas de manejo avaliada pela dinâmica da matéria orgânica e atributos relacionados. R. Bras. Ci. Solo, 29:777-788, 2005.

DEBIASI, H.; LEVIEN, R.; TREIN, C.R.; CONTE, O. \& MAZURANA, M. Capacidade de suporte e compressibilidade de um Argissolo, influenciadas pelo tráfego e por plantas de cobertura de inverno. R. Bras. Ci. Solo, 32:2629-2637, 2008.

DIAS JUNIOR, M.S. \& PIERCE, F.J. O processo de compactação do solo e sua modelagem. R. Bras. Ci. Solo, 20:175-182, 1996.

EMPRESA BRASILEIRA DE PESQUISAAGROPECUÁRIA EMBRAPA. Sistema brasileiro de classificação de solos. Brasília, Embrapa Produção de Informação, 2006. 412p.

EMPRESA BRASILEIRA DE PESQUISA AGROPECUÁRIA EMBRAPA. Manual de métodos e análise de solo. 2 ed. Rio de Janeiro: CNPS, 1997. 212 p.

HAMZA, M.A. \& ANDERSON, W.K. Soil compaction incropping systems: A review of the nature, causes and possible solutions. Soil Till. Res., 82:121-145, 2005.

HSIAO, T.C. \& XU, L.K. Sensitivity of growth of roots versus leaves to water stress: biophysical analysis and relation to water transport. J. Exp. Bot., 51:1595-1616, 2000.

IMHOFF, S. Indicadores de qualidade estrutural e trafegabilidade de Latossolos e Argissolos Vermelhos. Piracicaba, Escola Superior de Agricultura "Luiz de Queiroz”, Universidade de São Paulo, 2002. 94p. (Tese de Doutorado)

KARLEN, D.L.; MAUSBACH, M.J.; DORAN, J.W.; CLINE, R.G.; HARRIS, R.F. \& SCHUMAN, G.E. Soil quality: A concept, definition, and framework for evaluation. Soil Sci. Soc. Am. J., 61:4-10, 1997.

LAL, R. \& SHUKLA, M.K. Principles of soil physics. Ohio, 2005. 699p.

LIBARDI, P.L \& JONG van LIER, Q. Atuação dos fatores físicos do solo no desenvolvimento do sistema radicular. In: FERNANDES, M.F.; TAVARES, E.D. \& LEAL, M.L., eds. WORKSHOP SOBRE SISTEMA RADICULAR: Metodologias e estudo de casos. Aracaju, Embrapa Tabuleiros Costeiros, 1999. p.47-56. 
MAZURANA, M. Atributos físicos, mineralógicos e matéria orgânica de solos relacionados à capacidade de suporte de carga. Porto Alegre, Universidade Federal do Rio Grande do Sul, 2011. 169p. (Dissertação de Mestrado)

O'SULLIVAN, M.F.; HENSHALL, J.K. \& DICKSON, J.W. A simplifid method for estimating soil compaction. Soil Till. Res., 49:325-335, 1999.

REICHERT, J.M.; REINERT, D.J. \& BRAIDA, J.A. Qualidade dos solos e sustentabilidade de sistemas agrícolas. R. Ci. Amb., 27:29-48, 2003.

SCHÄFFER, B.; ATTINGER, W. \& SCHULIN, R. Compaction of restored soil by heavy agricultural machinery - Soil physical and mechanical aspects. Soil Till. Res., 93:28-43, 2007.

SEQUINATTO, L. Qualidade física do solo e rendimento de grãos num Argissolo em processo de recuperação. Porto Alegre, Universidade Federal do Rio Grande do Sul, Porto Alegre, 2010. 141p. (Tese de Doutorado)
SILVA, A.P.; KAY, B.D. \& PERFECT, E. Characterization of the least limiting water range. Soil Sci. Soc. Am. J., 58:1775-1781, 1994.

SOUZA, E.D.; CARNEIRO, M.A.C.; PAULINO, H.B.; SILVA, C.A. \& BUZETTI, S. Frações do carbono orgânico, biomassa e atividade microbiana em um Latossolo Vermelho sob cerrado submetido a diferentes sistemas de manejos e usos do solo. Acta Sci. Agron., 28:323-329, 2006

ZHAO, Y.; PETH, S.; HORN, R.; KRÜMMELBEIN, J.; KETZER, B.; GAO, Y.; DOERNER, J.; BERNHOFER, C. \& PENG, X. Modeling grazing effects on coupled water and heat fluxes in Inner Mongolia grassland. Soil Till. Res., 109:75-86, 2010.

VEZZANI, F.M. \& MIELNICZUK, J. O solo como sistema. Curitiba, 2011. 104p.

VEIGA, M.; HORN, R.; REINERT. D.J. \& REICHERT, J.M. Soil compressibility and penetrability of an Oxisol from Southern Brazil, as affected by long-term tillage systems. Soil Till. Res., 92:104-113, 2007. 